Conclusions Over one third of Blacks aged 65 and older never underwent cervical cancer screening, and the rate of non-compliant screening is increasing. With the highest incidence of cervical cancer in Blacks seen in this age group, the role of individualized cervical cancer screening guidelines should be considered.

\section{EPV057/\#294 DEVELOPMENT OF A LARGE SWINE MODEL FOR ANATOMICAL AND FUNCTIONAL ASSESSMENT OF THE FEMALE PELVIC AUTONOMIC NERVES IN WOMEN}

'J Mun, '1 Kim, ${ }^{2}$ S Lee, ${ }^{1} \mathrm{HS}$ Kim, ${ }^{1} \mathrm{~J}-\mathrm{W}$ Kim. ${ }^{1}$ Seoul National University College of Medicine, Department of Obstetrics and Gynecology, Seoul, Korea, Republic of; ${ }^{2}$ Keimyung University School of Medicine, Department of Obstetrics and Gynecology, Seoul, Korea, Republic of

\subsection{6/ijgc-2021-IGCS.125}

Objectives The anatomy and function of the pelvic autonomic nerves are not yet fully understood despite the development of nerve-sparing radical surgery for cervical cancer. Thus, we developed a female animal model for anatomical and functional assessment of the pelvic autonomic nerves in women.

Methods We used eight female swine models weighing about $30 \mathrm{~kg}$ each and assessed the anatomy of their pelvic autonomic nerves. We also evaluated the nerves' function by measuring the pressure of the bladder, vagina, and rectum after electrically stimulating the parasympathetic nerves with or without resection of the sympathetic nerves.

Results Three swine models were dissected for anatomical assessment and showed similar patterns. Although there were some anatomic variations, most showed identical pathways of the sympathetic and parasympathetic nerves that eventually led to the formation of superior and inferior hypogastric nerves respectively, as well as the individual branches of the pelvic plexus. The remaining eight models were used for functional assessment. Before resection of the sympathetic nerves, stimulation of parasympathetic nerves showed increased interval to contraction and duration of contraction but decreased maximal contractile pressure and frequency in the pelvic organs, while results revealed the contrary after resection of the sympathetic nerves.

Conclusions We were able to identify the anatomy and function of pelvic autonomic nerves in swine models and found them to be similar to those of women. Further studies should be done to compare the two in order to master the knowledge of female pelvic autonomic nerves.

\section{EPV058/\#315 ASSOCIATION OF HLA-G POLYMORPHISMS WITH HIGH-RISK HPV+ CERVICAL PATHOLOGIES SUSCEPTIBILITY}

${ }^{1}$ I Zidi, ${ }^{1,2}$ | Zemni*, ${ }^{1} \mathrm{~K}$ Tizaoui, ${ }^{1} \mathrm{HI}$ Ouzari, ${ }^{1,3} \mathrm{~N}$ Boujelbene. ${ }^{1}$ Sciences Faculty of Tunis, University of Tunis El Manar, Laboratory of Microorganismes and Active Biomolecules, Tunis, Tunisia; ${ }^{2}$ Salah Azaiez Institute of Cancerology, University of Tunis El Manar, Department of Surgical Oncology, Tunis, Tunisia; ${ }^{3}$ Salah Azaiez Institute of Cancerology, University of Tunis El Manar, Department of Pathology, Tunis, Tunisia

\subsection{6/ijgc-2021-IGCS.126}

Objectives HLA-G gene polymorphisms have been linked to many cancers particularly to cervical squamous cell carcinoma (CSCC). In this meta-analysis, we studied the association of
HLA-G +3142 C/G and 14bp Insertion/deletion (Ins/Del) polymorphisms with cervical pathologies susceptibility.

Methods A comprehensive systematic literature search in Medline, Pubmed, Cochrane, Embase, and Web of Science databases was performed to look up relevant studies. We identified four studies for HLA-G +3142 C/G (299 patients with HPV+ high-risk cervical pathologies and 870 healthy controls (HC)); and six studies for HLA-G14bp Ins/Del (693 patients with HPV+ high-risk cervical pathologies and 2536 $\mathrm{HC})$. The association was studied through the calculation of the odds ratio (OR) and the corresponding 95\% confidence interval (CI).

Results HLA-G +3142 C/G polymorphism and HLA-G $14 \mathrm{bp}$ Ins/Del significantly enhanced the risk for $\mathrm{HPV}+$ cervical pathologies only in Asians conversely to overall population and Caucasians. HLA-G $+3142 \mathrm{C} / \mathrm{G}$ enhanced the HPV+ high-risk cervical pathologies risk under allelic $\mathrm{C}$ vs. $\mathrm{G}$ model $(\mathrm{OR}=1.321,95 \mathrm{CI} \%=1.035-1.686, \mathrm{p}=0.025)$ and under the genotypic model $\mathrm{CC}$ vs. $\mathrm{GG}+\mathrm{GC} \quad(\mathrm{OR}=2.028, \quad 95 \mathrm{CI} \%$ $=1.337-3.075, \mathrm{p}=0.001)$. HLA-G 14bp Ins/Del increased also the $\mathrm{HPV}+$ cervical pathologies risk only under the genetic model (InsIns vs. DelDel+InsDel) $(\mathrm{OR}=1.910,95 \% \mathrm{CI}=1.151$ 3.171, $\mathrm{p}=0.012$ ) in Asians.

Conclusions Our preliminary meta-analysis showed a significant association of HLA-G $+3142 \mathrm{C} / \mathrm{G}$ polymorphism and HLA-G 14bp Ins/Del with HPV+ high-risk cervical pathologies susceptibility in Asians. Further studies still needed in other ethnicities to clearly establish our findings.

\section{EPV059/\#324 INTERACTIVE FLASHCARDS IMPROVE HUMAN PAPILLOMAVIRUS VACCINE KNOWLEDGE AND WILLINGNESS TO RECOMMEND AMONG NURSES}

${ }^{1}$ SOA Leung*, ${ }^{2} E$ Duffey-Lind, ${ }^{3} \mathrm{~K}$ Welch, ${ }^{1} \mathrm{~S}$ Feldman. 'Brigham and Women's Hospital, Obstetrics and Gynecology, Boston, USA; '2Dana Farber Cancer Institute, Pediatric Oncology, Boston, USA; ${ }^{3}$ Team Maureen, Team Maureen, North Falmouth, USA

\subsection{6/ijgc-2021-IGCS. 127}

Objectives The Human Papillomavirus (HPV) vaccine prevents cervical and other HPV-associated cancers by preventing infection with oncogenic HPV subtypes. In the United States, only $57 \%$ of women and $53 \%$ of men in the recommended age groups have received all recommended doses. Our objective was to create a 7-minute interactive learning platform to improve knowledge of HPV and to assess willingness to recommend the vaccine among nurses.

Methods Pre- and post-intervention questions on HPV-associated cancers, vaccine eligible groups, dosing schedules, adverse events, and willingness to recommend the vaccine were posed. The intervention consisted of 10 flashcards in a questionanswer format with up-to-date information and responses to frequently asked questions (e.g., Who should receive the vaccine and how is it given? Some parents may worry that their child will think that getting this vaccine makes it OK to have sex, how do I answer?).

Results All 113 participants (40.9 \pm 11.6 years-old, 58\% with $>10$ years in practice) identified cervical cancer as an HPVassociated cancer. Post-intervention, there was improvement in recognition of other $\mathrm{HPV}$-associated cancers (70\% to $94 \%)$ and knowledge of dosing schedule (46\% to 93\%). $7 \%$ versus $1.7 \%$ of participants agreed with unproven adverse events pre- and post-intervention. 94\% of participants strongly agreed that they would recommend the HPV vaccine to patients and 